\title{
BEMPU Bracelet: Potentially Useful But Still Requires Robust Validation
}

\author{
RAJA ASHOK KogantI AND ASHOK DEORARI* \\ Neonatal Division, Department of Pediatrics, \\ All India Institute of Medical Sciences, New Delhi 110029. \\ *ashokdeorari_56@hotmail.com
}

$\mathrm{T}$ hermal protection in neonates is one of the four basic elements of essential newborn care and is critical for newborn survival [1]. Hypothermia directly or indirectly has been implicated in the causation of deaths of neonates, thereby prompting the development of a variety of devices and tools for its early detection and timely management. These tools range from the simple 'human touch' to the automated hypothermia detection devices (AHDD). In the heterogeneous social and health systems in India, a simple, affordable and locally acceptable tool is needed to detect hypothermia and alert care providers.

Sharma, et al. [2] in this issue of Indian Pediatrics, present the findings of a clinical study about the effectiveness of one such device, the BEMPU bracelet. A previously published study on the same device was conducted in a tertiary-care hospital, which had round the clock nursing backup [3]. Promising results were reported with the reported sensitivity and specificity of over $95 \%$ to detect hypothermia; although, the diagnostic accuracy for different severity of hypothermia was not reported. The present study [2] is another step in evaluating the role of AHDD in community settings, and reports significant reduction in neonatal mortality in the intervention group. However, the results need to be interpreted carefully in the context of the caveats discussed below.

The direct attribution of hypothermia as a cause of neonatal mortality may not be true. Hypothermia and death in neonates indeed have a strong association but causality can not be established in majority of the cases. Hypothermia may be a consequence rather than cause of neonatal illnesses (e.g. sepsis) in many cases. This relative lack of clarity is further compounded by the existence of weak vital statistics in developing countries because of which outcome of a large number of enrolled neonates is not known. Undoubtedly, early detection of hypothermia plays an important role in limiting morbidity, but relating all instances of hypothermia to deaths that can be prevented by the device may overrate the effectiveness of the device.
This pilot trial conducted in Rajasthan [2] does illustrate the feasibility and acceptability of using the device at the community level; however, the families included in the trial were also provided regular weekly follow-up visits and reminder phone calls, which are potent co-interventions influencing mortality. While the device is designed in attractive colors, resembles a mini version of a modern smartwatch and emits multicolored lights signifying normo- and hypo-thermia, it is uncertain whether the illiterate tribal population can interpret these cues. The single-use device is priced at INR 2499 and once activated has a shelf life of 4 weeks. Affordable cost is the key to accessibility in developing countries [4], and the 'prohibitive' cost of the device may be a major barrier for its widespread use in the public sector. The authors themselves opine that in the context of India's limited resources and to limit wasteful health expenditure, it is important to establish the cost-effectiveness of this device before its scaling up. The innovators should work to make this device as multi-use, which can not only make it affordable but also reduce its environmental footprint.

The authors [2] claim a positive behavior change in the parents of enrolled neonates as evident by higher follow-up rates at four weeks (59\%) in the study group; however, the observed effect could be a consequence of performance bias wherein the study group has been given more attention. Performance bias is an inherent drawback of unblinded trials, and a well-conducted study with robust design and blinding (of the person making phone calls/home visits) could address this issue in future. The study also included neonates with malformations; it is also unclear as to how the causes of death were assigned to neonates.

A simple yet cost-effective method to detect hypothermia is by using the hand-touch method. Palpation of the forehead, abdomen and foot [5] has shown a reasonable sensitivity in the detection of hypothermia. Training mothers and healthcare workers in this skill is an effective method in a resource-limited setting. Any future study should include the 'touch 
method' of detecting hypothermia in the comparison arm thus evaluating the incremental benefit of the device.

Lastly, it is important to realize that an AHDD may be used as a tool to detect hypothermia that triggers corrective and preventive action like kangaroo mother care (KMC) and a hospital visit. It is just one component of care - we should not lose sight of the fact that only holistic care including optimum feeding practices, promoting $\mathrm{KMC}$, hygiene, ensuring follow up care, and empowering health workers and mothers in detecting signs of severe illness for early care-seeking is going to save neonatal lives [6].

Finding: None; Conflict of interest: None stated.

\section{REFERENCES}

1. Maternal and newborn health safe motherhood. World Health Organization: Essential newborn care. Report of a technical working group: Geneva, 1996.

2. Sharma M, Morgan V, Siddadiah M, Songara D, Bhawsar $\mathrm{RD}$, Srivastava A. Impact of a novel hypothermia alert device on death of low birthweight babies at four weeks: A non-randomized controlled community-based trial. Indian Pediatr. 2020;57:305-9.

3. Tanigasalam V, Vishnu Bhat B, Adhisivam B, Balachander B, Kumar H. Hypothermia detection in low birth weight neonates using a novel bracelet device. J Matern Fetal Neonatal Med. 2019;32:2653-6

4. Shaw KM. Leveraging affordable innovation to tackle India's healthcare challenge. IIMB Management Review. 2018;30:37-50.

5. Singh M, Rao G, Malhotra AK, Deorari AK. Assessment of newborn baby's temperature by human touch: A potentially useful primary care strategy. Indian Pediatr. 1992;29:449-52.

6. Young Infants Clinical Signs Study Group. Clinical signs that predict severe illness in children under age 2 months: A multicentre study. Lancet. 2008;371:135-42. 